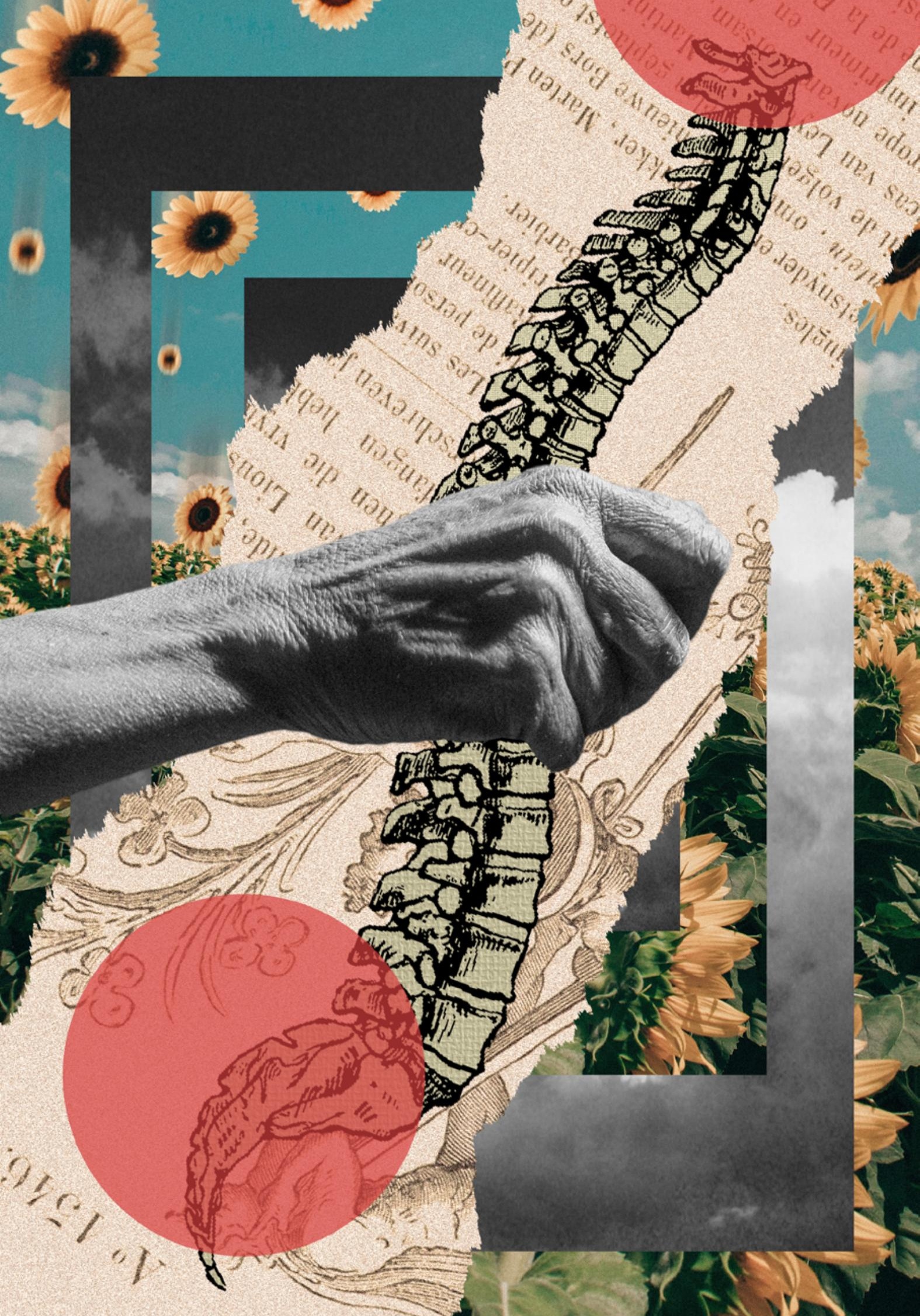




\title{
Intervenção fisioterapêutica na promoção e prevenção de distúrbios da coluna vertebral em idosos: um relato de experiência
}

\author{
Physiotherapeutic intervention in the promotion and prevention of disturbances of the \\ vertebral column: a report of experience
}

André Eduardo Falcoski Doliny Fisioterapeuta graduado na Universidade Federal do Paraná - UFPR andredoliny@gmail.com

Flávia Cristina Miranda Estudante do curso de Fisioterapia da Universidade Federal do Paraná - UFPR flacmiranda@gmail.com

Jéssica Perez Estudante do curso de Fisioterapia da Universidade Federal do Paraná - UFPR perez.je147@gmail.com

Luiza Grassmann Dias Estudante do curso de Fisioterapia da Universidade Federal do Paraná - UFPR luizagrassmann@gmail.com

Ana Carolina Brandt de Macedo Doutora, Departamento de prevenção e promoção em Fisioterapia. Universidade Federal do Paraná - UFPR acbrandtmacedo@gmail.com

Ariani Szkudlarek Doutora, Departamento de prevenção e promoção em Fisioterapia. Universidade Federal do Paraná - UFPR arianiinaira@hotmail.com

Rubneide Gallo Doutora, Departamento de prevenção e promoção em Fisioterapia. Universidade Federal do Paraná - UFPR rubneidegallo@gmail.com

Talita Gnoato Zotz Doutora, Departamento de prevenção e promoção em Fisioterapia. Universidade Federal do Paraná - UFPR

\section{RESUMO}

Este relato de experiência tem como objetivo expor a vivência de alunos da Universidade Federal do Paraná - UFPR na disciplina de Habilidades Fisioterapêuticas, os quais montaram um grupo de promoção e prevenção a doenças de coluna vertebral em idosos. Foram realizados 13 encontros com média de 3 horas cada em um espaço cedido, localizado no bairro Alto no municipio de Curitiba - PR. No local, foram oferecidas palestras educacionais com temas sobre a prevenção de distúrbios musculoesqueléticos e da coluna vertebral; informações sobre alimentação: respiração; depressão; risco de quedas; e a importância de exercícios físicos. Associadas aos temas, eram planejadas atividades lúdicas que permitiram um melhor aprofundamento e consolidação do assunto abordado. Os participantes eram estimulados a interagirem com suas histórias e experiências. Observa-se que as atividades do grupo obtiveram resultados significantes tanto para os alunos quanto para os idosos. Esses puderam estabelecer um vinculo firmado pelos encontros semanais. Além dos beneficios da atividade física inserida regularmente na vida dos idosos, o grupo proporcionou a interação social entre as diversas pessoas que frequentaram os encontros. Foi visto, com as situações vivenciadas na disciplina, que existe uma necessidade de viabilizar programas com foco em educação em saúde que proporcionem à população um envelhecimento mais saudável e menos oneroso. Por fim, concluímos que a atuação do fisioterapeuta na saúde primária é de grande importância para a população idosa. Esta prática minimiza possiveis acometimentos causados pela senescência. Considera-se que o objetivo do grupo foi alcançado visto o crescimento do número de participantes a cada novo encontro e por meio dos relatos sobre a melhora da qualidade de vida desses.

Palavras-chave: Idoso; Atenção primária à saúde; Saúde pública; Relato de experiência; Fisioterapia.

\section{ABSTRACT}

This study aims to expose the experiences of physical-therapy students, of the Federal University of Paraná, in their discipline, who set up a group of promotion and prevention on spine diseases in elderly. A total of 13 meetings in bairro alto, Curitiba city were done, with an average of 3 hours each, where educational lectures were given on the prevention of musculoskeletal and spinal disorders, information on feeding, breathing, depression, risk of falls and the importance of physical exercise. With the themes were planned ludic activities that allow a better understanding and learning of the subject. Participants were encouraged to interact with their stories and experiences. It is observed that the implantation of the group had significant results for the students and the elderly, who established a bond In addition to the benefits of physical activity regularly implemented in the lives of the elderly, the group provided social interaction among people who attended it. It was seen, with the situations experienced in the discipline, that there is a need to promote programs focused on health education and provide the population with a healthier and less costly aging. Finally, we conclude that the physiotherapist's performance in primary health is of great importance for the elderly population, minimizing possible senescence effects and it is considered that the goal of the group was achieved with increasing number of participants at each new meeting and with the reports of improvement of the participants.

Keywords: Elderly. Primary Health Care. Public Health. 


\section{CONTEXTUALIZAÇÃO}

O perfil demográfico brasileiro tem demonstrado clara tendência ao envelhecimento. Entre 2005 e 2015, a proporção de idosos de 60 anos ou mais no pais passou de $9,8 \%$ para $14,3 \%$ (IBGE, 2015).

Com o aumento do número de idosos na população, os problemas gerados pelo processo de envelhecimento consequentemente também crescem. Dentre esses processos encontram-se a diminuição da elasticidade dos tecidos moles; articulações mais rigidas e menos capazes de absorver pressões; cartilagens menos elásticas; musculatura enfraquecida, levando à desaceleração dos movimentos e à perda da coordenação; os ossos podem se tornar osteoporóticos, ocasionando dificuldades com o equilibrio e alterações na postura corporal (Souza et al., 2019).

Segundo censo do IBGE de 2015, os idosos na faixa etária de 60 a 79 anos possuem como principal problema as doenças de coluna, as quais incluem o aumento da curvatura cifótica da coluna torácica e a diminuição da lordose lombar. As patologias em questão podem ser evitadas com projetos de Educação e Saúde, que também colaboram com a formação efetiva do modelo assistencial e reduzem os gastos públicos com o atendimento nas atenções secundárias e terciárias (Maziero et al., 2018). A atuação do fisioterapeuta dentro da atenção primária em saúde não se limita a um papel reabilitador. Sua função como agente garante a integralidade e a resolução dos problemas na população na qual está inserido (Castro, Cipriano \& Martinho, 2006).

O envelhecimento geralmente vem acompanhado da fragilização do idoso nos niveis biopsicossociais, levando a uma diminuição na capacidade adaptativa do idoso frente às novas vivências. Este fato caracteriza transformações no estilo de vida, tornando assim o idoso dependente do ambiente familiar, o que possivelmente influenciará em sua qualidade de vida (Schenker et al., 2019).

A qualidade de vida na velhice é dependente de muitos elementos e é fundamental para garantir maior longevidade. Ela depende das condições biológicas, das condições físicas do ambiente, das condições oferecidas pela sociedade - relativas à renda, saúde, educação -, do grau de urbanização, das condições de trabalho (Almeida et al., 2006).

Tendo em vista o aumento da população idosa e o consequente o aumento de morbidades associadas, faz-se necessária uma abordagem de melhor qualidade visando à prevenção de doenças crônicas não transmissiveis e a promoção da saúde. Este trabalho tem por objetivo expor o relato de experiência de um grupo de educação em saúde, que teve como propósito prevenir distúrbios da coluna vertebral em idosos a partir da realização de atividades lúdicas realizadas em um espaço cedido, localizado no bairro Alto no município de Curitiba - PR. 


\section{DESCRIÇÃO DA EXPERIÊNCIA}

Foram promovidos, pelos alunos graduandos do $4^{\circ}$ período do curso de Fisioterapia da Universidade Federal do Paraná, encontros sob a orientação da Professora Dra. Ana Carolina Brandt de Macedo. Foram, ao total, 13 encontros, realizados de agosto a novembro de 2019, os quais se davam todas as segundas-feiras com duração de, em média, 3 horas.

A população-alvo das ações foram idosos que moravam no Bairro Alto ou que eram atendidos na Unidade Básica de Saúde do Bairro Alto (UBS - Bairro Alto). A abordagem ocorreu através de contatos com os participantes do grupo do ano anterior, via telefone, também por meio de folders entregues no bairro e de cartazes fixados dentro da UBS.

Foram realizadas palestras educacionais com assuntos que abordavam a prevenção de distúrbios musculoesqueléticos e da coluna vertebral em idosos, além de informações sobre alimentação, respiração, depressão, risco de quedas e a importância de exercícios físicos. Associadas aos temas, eram planejadas atividades lúdicas que permitiram um melhor aprofundamento e consolidação do assunto abordado, visando à educação, melhora do bem estar, funcionalidade e a promoção da saúde em idosos.

A cada encontro, fez-se a aferição da pressão arterial de cada idoso participante. Em seguida, era realizada uma introdução teórica acerca do tema em questão, conduzida por um líder, permitindo aprendizado por parte dos participantes e possibilitando avaliá-los acerca de seus conhecimentos prévios. Para recolher as informações trabalhadas, foram confeccionados alguns cadernos, nos quais era fixado, no início do encontro, um resumo do tema do dia. Além disso, foi permitido que os participantes fizessem suas considerações (Quadro 1).

Quadro 1

\begin{tabular}{|c|c|c|}
\hline ENCONTRO & TEMA & ATIVIDADE \\
\hline 1 & $\begin{array}{l}\text { Anatomia da } \\
\text { coluna vertebral }\end{array}$ & $\begin{array}{l}\text { Durante a palestra, para uma melhor visuali- } \\
\text { zação do que é o disco intervertebral, foram } \\
\text { utilizados chicletes (Bubbaloo) para de- } \\
\text { monstrar o anel fibroso e o núcleo pulposo. } \\
\text { Para a atividade, foi elaborado um quiz } \\
\text { com perguntas sobre a palestra realizada. } \\
\text { Os participantes foram divididos em } \\
\text { dois grupos a fim de tornar a atividade } \\
\text { mais dinâmica e interativa. Cada par- } \\
\text { ticipante teve sua vez para responder, } \\
\text { no entanto, o grupo podia ajudar. } \\
\text { Caso uma pessoa tivesse acertado } \\
\text { e a outra errado, aquela que acertou } \\
\text { tinha o direito de dar uma prenda para } \\
\text { a que errou. A prenda poderia ser pintar } \\
\text { a outra pessoa com maquiagem } \\
\text { ou colocar peruca. }\end{array}$ \\
\hline
\end{tabular}




\begin{tabular}{|c|c|c|}
\hline 2 & $\begin{array}{l}\text { Relação entre atividade } \\
\text { física e prevenção de } \\
\text { distúrbios da coluna }\end{array}$ & $\begin{array}{l}\text { Relacionando com o tema da palestra } \\
\text { abordado no dia, realizaram-se coreo- } \\
\text { grafias de dança. As coreografias foram } \\
\text { demonstradas pelo lider e acompanhadas } \\
\text { pelos idosos. Foram realizadas três coreo- } \\
\text { grafias, sendo uma delas escolhida para } \\
\text { ser praticada até o final dos encontros. }\end{array}$ \\
\hline 3 & $\begin{array}{l}\text { Distúrbios da } \\
\text { coluna vertebral }\end{array}$ & $\begin{array}{l}\text { Foram elaborados dois quebra-cabeças } \\
\text { de colunas vertebrais. Uma com hiper- } \\
\text { cifose e retificação da lombar e outra } \\
\text { com escoliose. Os participantes foram } \\
\text { divididos em dois grupos. Esses deveriam } \\
\text { montar o quebra-cabeça e informar qual } \\
\text { o distúrbio presente na coluna vertebral. }\end{array}$ \\
\hline 4 & $\begin{array}{l}\text { A importância da inges- } \\
\text { tão de água mineral }\end{array}$ & $\begin{array}{l}\text { Após a palestra que expunha o quanto a } \\
\text { ingestão de água ajuda na saúde, foi feita } \\
\text { uma oficina de decoração de garrafinhas } \\
\text { de água mineral. A atividade teve o in- } \\
\text { tuito de fazer com que os participantes } \\
\text { personalizassem suas garrafas para } \\
\text { lembrarem de tomar a quantia adequada } \\
\text { de água durante o dia. Além disso, foram } \\
\text { propostos exercícios de propriocepção e } \\
\text { de dança com um X-box (videogame). }\end{array}$ \\
\hline 5 & $\begin{array}{l}\text { Alimentação relacionada } \\
\text { a distúrbios da coluna } \\
\text { vertebral e como a falta } \\
\text { de cálcio pode afetar } \\
\text { a sua integridade }\end{array}$ & $\begin{array}{l}\text { Foi realizada uma tarde de produção de } \\
\text { sucos naturais com base em receitas pré- } \\
\text { determinadas na palestra introdutória. Os } \\
\text { componentes dos sucos foram: goiaba, } \\
\text { maçã, banana, couve, gengibre, limão, } \\
\text { cenoura, beterraba, hortelã, maracujá e } \\
\text { laranja. Estes ingredientes foram proces- } \\
\text { sados sem adição de água e açúcar. }\end{array}$ \\
\hline 6 & $\begin{array}{l}\text { Consequência das } \\
\text { quedas relacionadas } \\
\text { à coluna vertebral }\end{array}$ & $\begin{array}{l}\text { Em função das quedas recorrentes causadas } \\
\text { por deslizes em tapetes, realizou-se uma } \\
\text { oficina onde foi ensinado aos participantes } \\
\text { a emborracharem as peças. Assim, as } \\
\text { quedas dentro das próprias casas seriam } \\
\text { evitadas. Além disso, foram passadas } \\
\text { dicas e informações sobre como adaptar } \\
\text { o próprio lar contra o risco de quedas. }\end{array}$ \\
\hline 7 & $\begin{array}{l}\text { Relação da postura com } \\
\text { a coluna vertebral }\end{array}$ & $\begin{array}{l}\text { Após a palestra, cinco voluntários realizaram } \\
\text { atividades, como: varrer, carregar sacolas, } \\
\text { sentar e levantar caixas. Em seguida, o grupo } \\
\text { fez uma avaliação de cada voluntário e pon- } \\
\text { tuou se as posturas adotadas para realizar } \\
\text { as atividades estavam adequadas. Também } \\
\text { foi promovida a dinâmica da "corrida do } \\
\text { ovo". Ganhou o grupo em que todos os par- } \\
\text { ticipantes carregaram o ovo com a postura } \\
\text { mais adequada até o final do percurso. }\end{array}$ \\
\hline 8 & $\begin{array}{l}\text { Respiração e crescimento } \\
\text { da coluna: isostretching }\end{array}$ & $\begin{array}{l}\text { A atividade realizada pelos participantes do } \\
\text { grupo neste dia foi vivenciar uma sequência } \\
\text { inicial de movimentos básicos do isostret- } \\
\text { ching, associada sempre com a respiração } \\
\text { que o próprio método propõe. O objetivo, } \\
\text { além, é claro, do fortalecimento dos mús- } \\
\text { culos intrinsecos da coluna, era de que eles } \\
\text { experimentassem uma postura mais global, }\end{array}$ \\
\hline
\end{tabular}




\begin{tabular}{|c|c|c|}
\hline & & $\begin{array}{l}\text { tendo uma melhor noção do próprio corpo. } \\
\text { E com o contato inicial com o método, } \\
\text { os interessados poderiam procurar um } \\
\text { grupo e dar prosseguimento à prática. }\end{array}$ \\
\hline 9 & $\begin{array}{l}\text { Fatores relacionados } \\
\text { à perda de equilibrio } \\
\text { e coluna vertebral }\end{array}$ & $\begin{array}{l}\text { Com o objetivo de promover exercícios } \\
\text { multissensoriais para o equilibrio, foi } \\
\text { elaborado um circuito proprioceptivo com } \\
13 \text { estações. Os exercicios consistem em } \\
\text { movimentos de cabeça, pescoço e olhos; } \\
\text { controle postural em várias posições (em } \\
\text { apoio bipodal e unipodal, com marcha de } \\
\text { frente, de costas, lateral, em flexão plantar e } \\
\text { calcanhar) e uso de superfície instável. As- } \\
\text { sociados ao circuito, foram realizados exer- } \\
\text { cícios de fortalecimento e alongamentos. }\end{array}$ \\
\hline 10 & $\begin{array}{l}\text { Importância dos jogos } \\
\text { lúdicos na funcionalidade } \\
\text { da coluna vertebral }\end{array}$ & $\begin{array}{l}\text { Foram realizadas atividades lúdicas e brin- } \\
\text { cadeiras, que abrangeram aspectos relacio- } \\
\text { nados com atividades do cotidiano deles, } \\
\text { demonstrando assim que esse "jogos" po- } \\
\text { dem auxiliar na realização das atividades de } \\
\text { vida diária dos idosos de uma maneira fun- } \\
\text { cional. Como por exemplo o volençol. Jogo } \\
\text { esse em que são formadas duas equipes e } \\
\text { uma passa a bola para a outra com o auxilio } \\
\text { de um lençol, trabalhando assim equilibrio } \\
\text { estático e dinâmico, ritmo, sincronia, trabalho } \\
\text { em equipe, coordenação membros inferiores } \\
\text { com membros superiores. Outro exemplo } \\
\text { foram as brincadeiras de morto vivo realizada } \\
\text { com cadeiras, e a própria dança da cadeira. }\end{array}$ \\
\hline 11 & $\begin{array}{l}\text { Depressão como doença } \\
\text { que afeta o emocional } \\
\text { e interfere na saúde } \\
\text { física do idoso }\end{array}$ & $\begin{array}{l}\text { Para promover uma maior interação } \\
\text { entre os idosos na atividade lúdica, foi } \\
\text { elaborado um bingo com } 14 \text { prêmios. Foi } \\
\text { entregue a cada participante uma cartela e } \\
\text { alguns grãos de feijão para marcação dos } \\
\text { números. "Batia" a cartela quem fechasse } \\
\text { linhas na diagonal, horizontal e vertical. }\end{array}$ \\
\hline 12 & $\begin{array}{l}\text { Revisão de temas } \\
\text { abordados }\end{array}$ & $\begin{array}{l}\text { Após a revisão de todos os temas abordados } \\
\text { ao longo dos encontros, foi aplicada uma } \\
\text { provinha para avaliação tanto da absorção } \\
\text { dos conhecimentos compartilhados quanto } \\
\text { para a verificação sobre a efetividade } \\
\text { da abordagem da equipe de alunos. }\end{array}$ \\
\hline 13 & Encerramento & $\begin{array}{l}\text { Como forma de despedida, organizou-se um } \\
\text { "amigo secreto" e uma confraternização. Em } \\
\text { roda, foi possivel que cada um expressasse } \\
\text { suas considerações acerca dos encontros. }\end{array}$ \\
\hline
\end{tabular}

Após cada atividade, era feito um alongamento com o objetivo de promover o encerramento das atividades. Além disso, ao final de cada atividade, a pressão dos participantes era aferida novamente.

Durante todos os encontros, era priorizado que os idosos interagissem durante as palestras, expondo suas experiências, sendo instigados a mostrarem suas dúvidas e fazendo-os participar de cada atividade com o intuito de que aprendessem mais sobre o assunto tratado no dia. Além disso, essa interação proposta fez com fosse criado um vínculo entre os discentes e os participantes 
e, a cada atividade, todos se sentiam mais à vontade para compartilhar as histórias e participar mais ativamente de todas as atividades propostas pelo grupo. A criação do vínculo e de um ambiente no qual todos se sentiam à vontade para expor qualquer dúvida, fez com que a adesão do grupo aumentasse de 8 para 20 participantes ao decorrer de todo o semestre de trabalho, formando um grupo muito heterogêneo. Fato esse que proporcionou aos discentes e aos participantes um maior e mais rico aprendizado.

\section{DISCUSSÃO}

A Fisioterapia, na atenção primária, vem trabalhando para propor um desenho assistencial à população, baseando-se na construção de estratégias que possibilitem suas diversas formas de atuação, promovendo ações de promoção e prevenção em saúde, como os grupos de atividade física e educação em saúde. Essas ações remodelam a visão de que o fisioterapeuta é um agente apenas reabilitador (Formiga \& Ribeiro, 2012).

Com a implantação da matéria de Habilidades Fisioterapêuticas, o fisioterapeuta foi visto, facilmente, como um agente implementador de saúde pelos participantes e não apenas como um reabilitador. As atividades possibilitaram aos participantes o acesso a diversas informações, novas experiências e também promoveram a efetividade da educação em saúde por meio da criação de uma rotina que inclui atividades físicas regulares.

A atividade física, quando planejada, contribui para a diminuição do sofrimento do idoso no âmbito biopsicossocial. Além disso, oferece a oportunidade da elevação da autoestima, da melhora das funções cognitivas e em quadros de depressão, também de prevenir o agravo de patologias comuns em idosos (Teixeira et al., 2016). Caso esse que pôde ser observado durante a palestra sobre depressão, na qual um dos participantes se viu dentro daquela realidade, pediu auxilio e foi encaminhado a um profissional.

Os comentários dos participantes sobre o desejo de que as atividades não chegassem ao fim foram repetidos. Segundo eles, a segunda-feira à tarde era um dos dias mais esperados da semana, que alegrava e proporcionava novos conhecimentos e interação entre eles mesmos e o grupo de alunos. Observa-se assim que as atividades promovidas pelo grupo obtiveram resultados extremamente significantes tanto para os idosos participantes quanto para os discentes envolvidos no projeto. Além dos benefícios da atividade física inserida regularmente na vida dos idosos, o grupo proporcionou a interação social entre as diversas pessoas que frequentaram os encontros.

As situações vivenciadas nesse relato reforçam a necessidade de viabilizar programas em que se promova a educação em saúde e proporcione à população um envelhecimento saudável e ativo. As ações promovidas são essenciais na prevenção do envelhecimento patológico, tornando esse processo menos oneroso. Vários participantes também relataram que estavam sentindo- 
se mais dispostos no dia a dia, realizando as atividades de vida diária com mais afinco e energia.

Considerando a aderência dos idosos, nota-se que o objetivo está sendo alcançado progressivamente. Observou-se um bom comprometimento dos idosos com as atividades propostas pelos alunos, tanto nas orientações teóricas quanto nas atividades práticas, o que mostra a efetividade do projeto. Durante as palestras, o interesse em querer saber, no aprender, era evidente, havendo uma troca mútua de conhecimentos entre os palestrantes e os participantes, gerando um laço mais forte do que simplesmente entre um instrutor e aprendizes, mas sim de amizade, com alegria, atividades físicas e conhecimento sendo partilhado no momento dos encontros.

No último encontro, foi feita uma roda com amigo secreto sorteado na hora, e todos tiveram a oportunidade de falar o que acharam dos encontros, o que estavam sentindo, sugestões ou críticas. Foi um momento que emocionou a todos, sem exceção, muitos não conseguiram segurar e algumas lágrimas apareceram, o sentimento que fica é de trabalho cumprido e de várias sementes plantadas nos corações de cada um, com lições para levar para o resto da vida, e se há uma frase para resumir todos esses momentos vividos ela é: "Nunca se é velho demais para aprender algo, e nem novo demais para ensinar, pois a vida nos oportuniza em todos os seus momentos uma troca contínua, basta saber em quais momentos você é mestre e em quais é preciso silenciar e ouvir."

\section{CONSIDERAÇÕES FINAIS}

O trabalho proporcionou uma grande troca de aprendizado e conhecimento entre os participantes e os alunos. Além disso, observou-se que a atuação do fisioterapeuta na saúde primária é de grande importância para a população idosa, minimizando possiveis acometimentos causados pela senescência. O êxito das atividades realizadas pode ser notado com o crescimento do número de participantes a cada novo encontro e espera-se que a principal ideia de ter um grupo de promoção e prevenção de saúde possa ser absorvida pelos participantes, assim como entender a sua importância. Também se nota que é necessário um maior investimento, por parte dos municípios, na criação de grupos que trabalhem com educação em saúde, trabalhando com a saúde em si e não apenas sobre uma patologia já instaurada. Pois o gasto com grupos de promoção e de prevenção de saúde é bem menor se comparado a futuros problemas que poderão ser apresentados caso essa prevenção não aconteça. Assim, fica evidente um melhor custo benefício. Essas ações promovem a saúde como um todo, gerando benefícios biopsicossociais aos participantes, auxiliando de maneira funcional na realização das atividades da vida diária. 


\section{REFERÊNCIAS}

Almeida, L. G. D., Leão, I. O., Oliveira, J. B., \& Santos, M. M. O. (2006). Promover a vida: uma modalidade da fisioterapia no cuidado à saúde de idosos na família e na comunidade. Revista Saúde.Com, 2 (1), 50-58.

Castro, S S., Cipriano Junior, G., \& Martinho, A. (2006, dez). Fisioterapia no programa de saúde da família: uma revisão e discussões sobre a inclusão. Fisioterapia em Movimento, 19(4), 55-62.

Instituto Brasileiro de Geografia e Estatística (IBGE). Recuperado em og de julho, 2017, de <http://www.ibge.gov.br>.

Formiga N. F., \& Ribeiro, K. S. (2012). Inserção do fisioterapeuta na atenção básica: uma analogia entre experiências acadêmicas e a proposta dos Núcleos de Apoio à Saúde da Familia (NASF). Revista Brasileira de Ciências da Saúde, 16(2), $113-122$

Teixeira, C. M., Nunes, F. M. S., Ribeiro, F. M. S., Arbinaga, F., \& Vasconcelos-Raposo, J. (2016). Atividade fisica, autoestima e depressão em idosos. Cuadernos de psicología del deporte, 16(3), 55-66.

Maziero, B. I., Da Cruz, C. D. G., Lima, E. G., Santiago, W., Szkudlarek, A. C., Zotz, T. G., GALLO, R. B. S., \& MACEDO, A. C. B. (2018, jan/jun). Prevenção de distúrbios musculoesqueléticos em idosos: relato de experiência. Extensão em Foco, 18, 6471.

Schenker, M., \& Costa, D. H. D. (2019). Avanços e desafios da atenção à saúde da população idosa com doenças crônicas na Atenção Primária à Saúde. Ciência \& Saúde Coletiva, 24, 1369-1380.

Sousa, H. J. S., FonteS, L. A. X., \& Oliveira, P. C. D. C. R. (2019). Análise da abordagem fisioterapêutica nas alterações senescentes e senis do sistema musculoesquelético. Revista da FAESF, 3(2).

Data de submissão: 31/01/2020

Data de aceite: 20/04/2020 